\title{
Enfoque práctico del control moderno
}

Con aplicaciones en Matlab

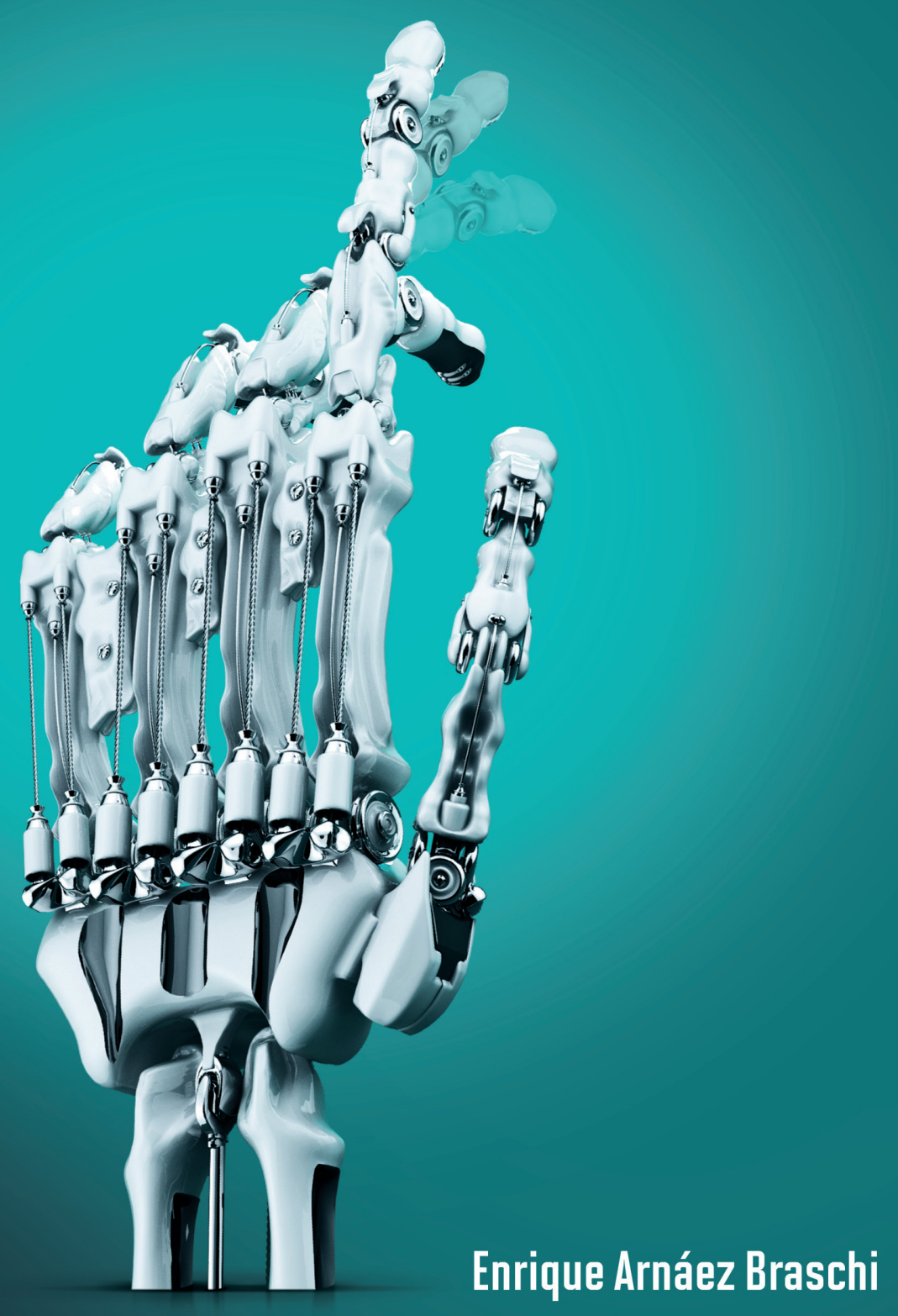





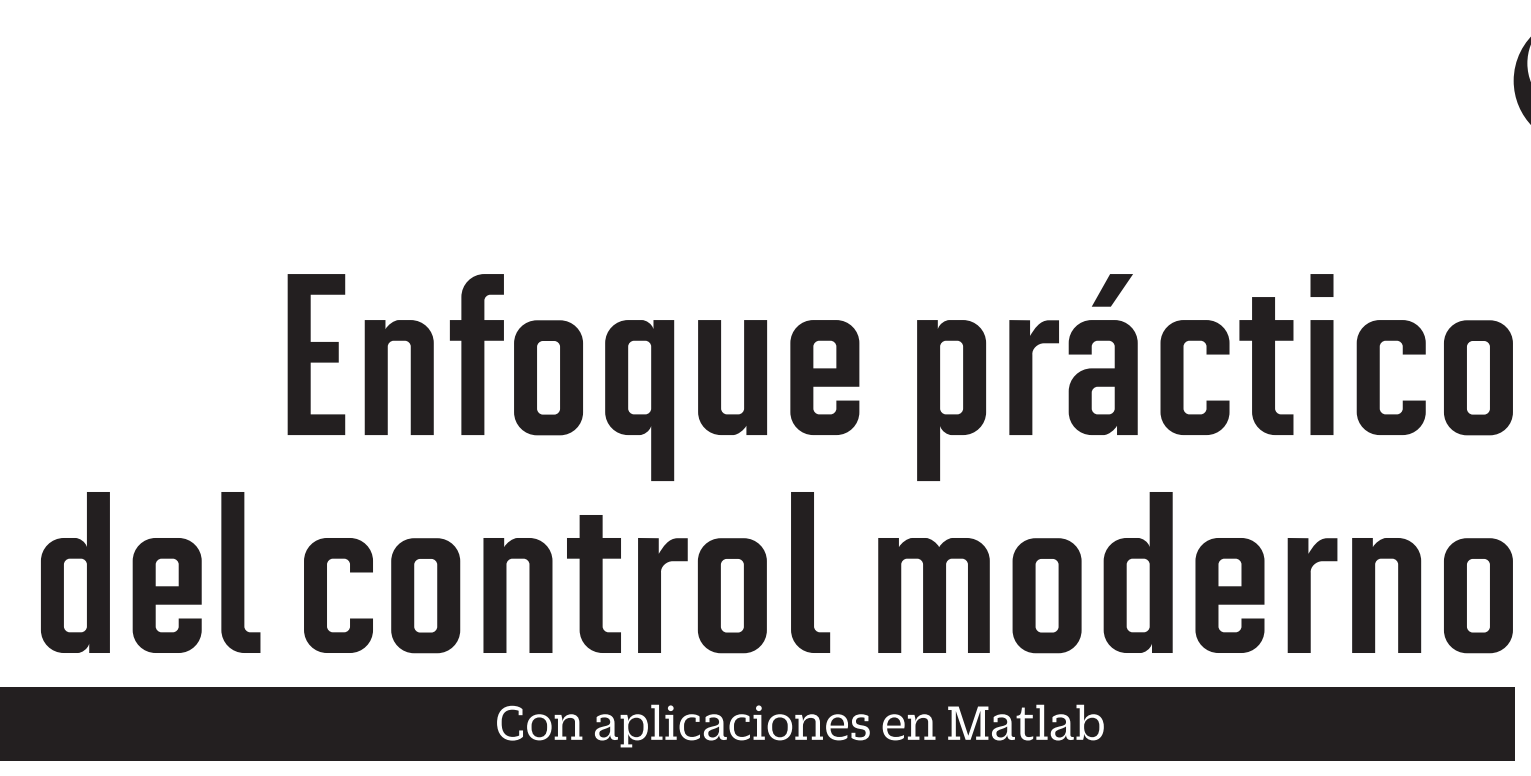

Lima, mayo de 2014

Universidad Peruana de Ciencias Aplicadas

Enrique Arnáez Braschi 
(C) Universidad Peruana de Ciencias Aplicadas (UPC)

Primera publicación: abril de 2014

Impreso en el Perú - Printed in Peru

Corrección de estilo: Silvana Velasco

Diseño de cubierta: Germán Ruiz Ch.

Diagramación: Otto Gonzales

Editor del proyecto editorial

Universidad Peruana de Ciencias Aplicadas S. A. C.

Av. Alonso de Molina 1611, Lima 33 (Perú)

Teléf: 313-3333

www.upc.edu.pe

Primera edición: mayo de 2014

Tiraje: 500 ejemplares

Este libro se terminó de imprimir en el mes de mayo de 2014, en los talleres gráficos de Metrocolor S. A., calle Los Gorriones 350, Chorrillos. Lima - Perú.

\section{Universidad Peruana de Ciencias Aplicadas (UPC) \\ Centro de Información}

Arnáez Braschi, Enrique. Enfoque práctico del control moderno: con aplicaciones en Matlab Lima: Universidad Peruana de Ciencias Aplicadas (UPC), 2014

ISBN: 978-612-4191-28-2

SISTEMAS DE CONTROL, CONTROL ELECTRÓNICO, CONTROLADORES, TRANSFORMACIONES MATEMÁTICAS 629.89 ARNA

Hecho el Depósito Legal en la Biblioteca Nacional del Perú Nº 2014-05721

Registro de Proyecto Editorial en la Biblioteca Nacional del Perú Nº 31501401400343

Todos los derechos reservados. Esta publicación no puede ser reproducida, ni en todo ni en parte, ni registrada en o transmitida por un sistema de recuperación de información, en ninguna forma ni por ningún medio, sea mecánico, fotoquímico, electrónico, magnético, electroóptico, por fotocopia o cualquier otro, sin el permiso previo, por escrito, de la editorial.

El contenido de este libro es responsabilidad del autor y no refleja necesariamente la opinión de los editores. 
Quiero agradecerles a mi esposa Mariella y a mis hijas Daniella y Andrea, por su apoyo y sacrificio al permitirme utilizar tantas horas que debieron ser para ellas, a mis padres por las enseñanzas que me dieron, a mis profesores y alumnos porque de todos ellos he aprendido permanentemente, a mis superiores, subalternos y amigos de la Marina de Guerra del Perú, por su permanente estímulo para continuar con los estudios e investigaciones emprendidas, a mis colegas tiradores, entrenadores y amigos del tiro deportivo por su respaldo y aliento permanente, y por sobre todo a Dios por su inmenso amor. 


\section{Contenido}

Introducción

\section{Capítulo 1: Introducción al control moderno}

$\begin{array}{ll}1.1 \text { Clasificación de los sistemas de control } & 18\end{array}$

$\begin{array}{ll}1.2 \text { Transformadas de Laplace } & 19\end{array}$

1.2.1 Propiedades de las Transformadas de Laplace 20

1.2.2 Expansión por fracciones parciales $\quad 20$

1.3 Álgebra de los diagramas de bloques 24

$\begin{array}{ll}1.4 \text { Estabilidad } & 31\end{array}$

\section{Capítulo 2: Análisis de la respuesta en el estado transitorio}

2.1 Sistemas de primer orden 35

2.2 Sistemas de segundo orden 44

2.2.1 Conceptos generales $\quad 52$

2.3 Diseño de controladores clásicos $\quad 58$

2.3.1 Tipos de controladores clásicos $\quad 59$

2.3.2 Ventajas y desventajas de los controladores clásicos 60

\section{Capítulo 3: Análisis de los sistemas de control en el dominio de la frecuencia}

3.1 Diagramas de Bode 66

$\begin{array}{ll}\text { 3.1.1 Estabilidad en frecuencia } & 71\end{array}$

3.1.2 Relación entre el tipo de sistema y los diagramas de magnitud-fase $\quad 72$

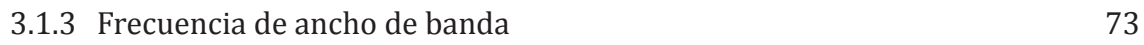

$\begin{array}{ll}\text { 3.1.4 Performance de lazo cerrado } & 76\end{array}$

3.2 Controladores o compensadores de adelanto o atraso de fase 77

$\begin{array}{ll}\text { 3.2.1 Compensador de adelanto de fase } & 78\end{array}$

$\begin{array}{ll}\text { 3.2.2 Compensador de atraso de fase } & 79\end{array}$

3.2.3 Compensador de adelanto-atraso de fase 80 


\section{Capítulo 4: Modelamiento matemático en espacio de estados}

4.1 Diseño en el espacio de estados 117

$\begin{array}{ll}\text { 4.2 Definiciones de espacio de estados } & 118\end{array}$

$\begin{array}{ll}4.2 .1 \text { Estado } & 118\end{array}$

4.2.2 Variables de estado 118

4.2.3 Vector de estado 118

$\begin{array}{ll}\text { 4.2.4 Ecuaciones de estado } & 118\end{array}$

$\begin{array}{ll}4.2 .5 \text { Espacio de estados } & 118\end{array}$

4.3 Pasos básicos para el modelamiento matemático 118

4.4 Programación en Matlab 131

4.5 Linealización de sistemas $\quad 135$

4.5.1 No linealidad al comienzo 136

4.5.2 No linealidad interna 137

4.5.3 No linealidad completa 138

4.6 Identificación práctica de un sistema de segundo orden 147

\section{Capítulo 5: Transformaciones}

5.1 Transformaciones de sistemas SISO 159

5.1.1 A partir de los coeficientes de una función de transferencia 159

a. Forma canónica controlable 159

b. Forma canónica observable 159

5.1.2 A partir de los polos de una función de transferencia 161

a. Forma canónica diagonal o modal 161

b. Forma canónica de Jordan 161

5.1.3 Comandos del Matlab para las transformaciones canónicas 163

5.2 Transformación de un sistema SIMO 166

5.3 Transformación de un sistema MIMO 167

$\begin{array}{ll}5.4 \text { Transformaciones inversas } & 167\end{array}$

5.4.1 Cálculo de la función de transferencia desde las formas canónicas 168

5.4.2 Cálculo de la matriz de transferencia a través de la Transformada $\begin{array}{ll}\text { de Laplace } & 170\end{array}$

5.5 Equivalencias o transformaciones de semejanza de las ecuaciones de estado 172

\section{Capítulo 6: Propiedades de los sistemas de espacio de estados}

$\begin{array}{ll}\text { 6.1 Solución de las ecuaciones de estado } & 177\end{array}$

6.1.1 Solución de las ecuaciones de estado de caso homogéneo 177

6.1.2 Matriz de transición de estados 178

6.1.2.1 Propiedades de la matriz de transición de estados 179 
6.1.3 Solución de las ecuaciones de estado de caso no homogéneo

6.2 Estabilidad en espacio de estados

6.3 Controlabilidad

6.3.1 Método para determinar la controlabilidad

6.3.2 Matlab para probar la controlabilidad

6.4 Observabilidad

6.4.1 Método para determinar la observabilidad

6.4.2 Matlab para probar la observabilidad

6.5 Controlabilidad de la salida

\section{| Capítulo 7: Diseño de controladores de estado}

7.1 Algoritmo para el cálculo del controlador de estados 200

$\begin{array}{ll}\text { 7.1.1 Método por excepción } & 201\end{array}$

7.2 Utilizando Matlab para el diseño de controladores 205

7.2.1 Matlab M-File de controlador.m para el diseño de controladores de estado

\section{| Capítulo 8: Diseño de observadores de estado}

8.1 Tipos de observadores de estado 230

8.2 Observadores de estado de orden completo 230

8.3 Diseño de observadores de estado 232

8.3.1 Algoritmo para el cálculo del observador de estados 233

8.3.2 Algoritmo de Ackerman 235

8.3.3 Método por excepción 236

8.4 Comparaciones con respecto al diseño de los controladores de estado 239

8.5 Utilizando el Matlab para el diseño de observadores de estado 241

8.6 Observador de estado en sistemas de lazo cerrado 242

$\begin{array}{ll}8.7 \text { Consideraciones adicionales } & 246\end{array}$

\section{Capítulo 9: Diseño de sistemas de seguimiento}

9.1 Tipos de sistemas de seguimiento 255

9.1.1 Sistema de seguimiento con integrador $\quad 255$

9.1.2 Sistema de seguimiento sin integrador 260

\section{| Capítulo 10: Control óptimo}

$\begin{array}{ll}10.1 \text { Criterio de estabilidad de Lyapunov } & 279\end{array}$

$\begin{array}{lll}\text { 10.1.1 Función definida positiva } & 279\end{array}$

10.1.2 Matriz definida positiva 280 
10.1.3 Función de Lyapunov 281

10.1.4 Prueba de estabilidad 281

10.1.5 Solución de la ecuación de Lyapunov 282

10.2 Control óptimo cuadrático 285

10.2.1 Optimización de parámetros mediante el criterio de Lyapunov 287

Apéndice: Introducción al Matlab

Operadores de matemáticas $\quad \mathrm{A}-2$

Operadores relacionales $\quad$ A-2

Operadores lógicos $\quad \mathrm{A}-3$

Variables $\quad$ A-3

Polinomios $\quad$ A-3

Condicional A-4

Lazos de programación $\quad$ A-4

Constantes del sistema $\quad$ A-5

Algunos comandos útiles $\quad$ A-6

Figuras y gráficos $\quad$ A-8

M-Files $\quad$ A-8

Toolboxes; Symbolic Math Toolbox (toolbox de matemáticas simbólicas) A-9

Integrales $\quad \mathrm{A}-12$

Derivadas $\quad$ A-13

Resolución de ecuaciones $\quad$ A-14

$\begin{array}{ll}\text { Transformaciones } & \text { A-15 }\end{array}$

Toolboxes de control y señales $\quad$ A-16

Funciones de transferencia de filtros analógicos $\quad$ A-22 


\section{Lista de ejemplos}

Ejemplo E.1.1: Expansión de fracciones parciales y cálculo de la Transformada inversa de Laplace

Ejemplo E.1.2: Expansión de fracciones parciales y cálculo de la Transformada inversa de Laplace

Ejemplo E.1.3: Expansión de fracciones parciales y cálculo de la Transformada inversa de Laplace

Ejemplo E.1.4: Determinación de la función de transferencia de sistema circuito RLC (entrada: voltaje/salida: corriente)

Ejemplo E.1.5: Determinación de la función de transferencia de sistema circuito RLC (entrada: voltaje/salida: caída de tensión en el capacitor)

Ejemplo E.1.6: Determinación de la función de transferencia de sistema masa-resorte

Ejemplo E.1.7: Determinación de la función de transferencia de sistema motor DC de posición

Ejemplo E.1.8: Cálculo de los polos y los ceros de una función transferencia

Ejemplo E.1.9: Cálculo de los polos y los ceros de una función transferencia

Ejemplo E.2.1: Análisis de un sistema de primer orden

Ejemplo E.2.2: Análisis de un sistema de primer orden con ruido en el sensor

Ejemplo E.2.3: Análisis de un sistema de segundo orden

Ejemplo E.2.4: Análisis de un sistema de segundo orden

Ejemplo E.2.5: Diseño de un controlador PID

Ejemplo E.3.1: $\quad$ Trazo de los diagramas de Bode

Ejemplo E.3.2: Determinación de la estabilidad de un sistema

Ejemplo E.3.3: Diseño de un controlador del péndulo invertido

Ejemplo E.3.4: Diseño de un compensador de adelanto de fase

Ejemplo E.3.5: Método de diseño de respuesta de frecuencia para el controlador de cabeceo de un avión

Ejemplo E.4.1: Cálculo de las ecuaciones de estado de un circuito RLC

Ejemplo E.4.2: Cálculo de las ecuaciones de estado de un sistema masa-resorte

Ejemplo E.4.3: Cálculo de las ecuaciones de estado de un sistema múltiple masa-resorte

Ejemplo E.4.4: Cálculo de las ecuaciones de estado de un sistema motor DC de posición o velocidad

Ejemplo E.4.5: Linealización de un sistema

Ejemplo E.4.6: Linealización de un sistema de suspensión de una bola por magnetismo

Ejemplo E.4.7: Linealización de un giróscopo

Ejemplo E.5.1: Obtención de las formas canónicas controlable, observable y diagonal de un sistema

Ejemplo E.5.2: Transformación de función de transferencia a espacio de estados

Ejemplo E.5.3: Transformación de función de transferencia a espacio de estados

Ejemplo E.5.4: Transformación de ecuaciones de estado en matriz de transferencia

Ejemplo E.5.5: Transformación de ecuaciones de estado en matriz de transferencia

Ejemplo E.5.6: Transformación equivalente de un sistema 
Ejemplo E.6.1: Determinación de la matriz de transformación de estados

Ejemplo E.6.2: Determinación de la respuesta en el tiempo de un sistema

Ejemplo E.6.3: Determinación de la estabilidad de un sistema

Ejemplo E.6.4: Determinación de la estabilidad de un sistema

Ejemplo E.6.5: Determinación de la controlabilidad de un sistema

Ejemplo E.6.6: Diseño de un controlador de estados para la altitud de un satélite

Ejemplo E.6.7: Determinación de la observabilidad de un sistema

Ejemplo E.6.8: Diseño de un observador de estados para los estados de un satélite

Ejemplo E.6.9: Determinación de la controlabilidad, observabilidad y controlabilidad de un sistema

Ejemplo E.6.10: Cálculo de las ecuaciones de estado y de las propiedades de un tren magnético

Ejemplo E.7.1: Determinación de un controlador por ubicación de polos por el método completo

Ejemplo E.7.2: Determinación de un controlador por ubicación de polos por el método por excepción

Ejemplo E.7.3: Determinación de un controlador por ubicación de polos

Ejemplo E.7.4: Determinación de un controlador por ubicación de polos, utilizando la función controlador.m en Matlab

Ejemplo E.7.5: Determinación de un controlador por ubicación de polos, utilizando la función controlador.m en Matlab

Ejemplo E.7.6: Prueba de la función controlador.m con un sistema MIMO

Ejemplo E.7.7: Problema del péndulo invertido

Ejemplo E.7.8: Diseño de un controlador de estados para un motor de posición

Ejemplo E.8.1: Determinación de la ecuación característica con un observador de estados

Ejemplo E.8.2: Determinación de un observador de estados

Ejemplo E.8.3: Determinación de un observador de estados

Ejemplo E.8.4: Determinar el valor de $l_{1}$ y $l_{2}$ desde el polinomio característico

Ejemplo E.8.5: Determinación de un observador de estados

Ejemplo E.8.6: Determinación del nuevo sistema de lazo cerrado con observador

Ejemplo E.8.7: Determinación de un observador de estados

Ejemplo E.8.8: Determinación de un observador de estados, utilizando la función acker en Matlab

Ejemplo E.8.9: Determinación de un observador de estados para un motor de posición

Ejemplo E.9.1: Diseño de un controlador de estados de seguimiento y determinación del sistema de lazo cerrado

Ejemplo E.9.2: Diseño de un controlador con acción integral

Ejemplo E.9.3: Diseño de un controlador de estados con acción integral para un motor de posición

Ejemplo E.9.4: Realimentar los estados con mediante un observador de estados para el motor de posición con controlador de acción integral

Ejemplo E.9.5: Realimentar los estados con mediante un observador de estados para el motor de posición con controlador de acción integral cuyo sensor genera ruido

Ejemplo E.9.6: Comparar y analizar las diferencias de la tercera variable de estado de cada uno de los casos presentados en los tres ejemplos anteriores 
Ejemplo E.10.1: Prueba de un función sobre si es definida positiva

Ejemplo E.10.2: Prueba de un función sobre si es definida positiva

Ejemplo E.10.3: Determinación de la matriz $P$ mediante la Ecuación de Riccati

Ejemplo E.10.4: $\quad$ Determinación de la matriz $P$ mediante la Ecuación de Riccati

Ejemplo E.10.5: Análisis de un sistema mediante el control óptimo

Ejemplo E.10.6: Diseño de un controlador de estados mediante la función de costos de control óptimo

Ejemplo E.10.7: Diseño de un controlador óptimo para un telescopio de instrucción

Ejemplo E.10.8: Diseño de un controlador óptimo para un telescopio de instrucción realimentando los estados mediante un observador

Ejemplo E.10.9: Análisis completo de control sobre un sistema diseñando todo tipo de controladores y cerrando el lazo con observadores 


\section{Introducción}

Este libro ha sido preparado pensando en condensar temas sumamente abstractos de manera sencilla que apoyen el dictado de los cursos relacionados, específicamente, me refiero a los temas de control moderno, ya que cuando me tocó aprender y luego dictar estos cursos, el lenguaje que empleaban las publicaciones y la forma de escribir las matemáticas eran sumamente complicadas.

Asimismo, no se tenían aplicaciones en Matlab de los ejemplos que planteaban, siendo una gran interrogante cómo los autores programaban y llegaban a los resultados.

En este libro se condensa, en una forma práctica, estudios, trabajos e investigaciones de más de catorce años tratando de plasmar el enfoque práctico de la parte teórica del control moderno.

La teoría de control moderno emplea durante sus diferentes etapas para el diseño de los controladores, un amplio número de ciencias y herramientas tales como álgebra lineal, teoría de vectores y matrices, cálculo diferencial y programación. Para esta última herramienta, empleamos el Matlab, por ello si el lector no está familiarizado con estos temas, es conveniente que primero desarrolle ciertas habilidades antes de comenzar con estos conocimientos, ya que solamente se mencionarán los procedimientos necesarios sin profundizar en ellos.

Adicionalmente, todo ingeniero que vaya a analizar el comportamiento de un sistema controlado, o para controlarlo, deberá investigar la teoría que sostiene dicho comportamiento. En este caso, usamos teoría de electricidad, electrónica, mecánica y dinámica de sólidos o fluidos, economía, química o cualquiera que fuera el o los campos de trabajo del sistema en cuestión.

Complementariamente, el control moderno utiliza análisis numérico, teoría de optimización, lógica difusa, redes neuronales y otras nuevas teorías que puedan mejorar el desempeño de los sistemas que manejemos.

Se puede ver que desde el Capítulo 1 al 3, abarcamos las áreas tradicionales del control clásico, tales como los análisis de la respuesta en el tiempo transitorio y en la frecuencia. Se presentan de manera completa y con ejemplos desarrollados, los conceptos fundamentales del control, ya que la teoría clásica permite hacerlo de una manera fácil de comprender.

Posteriormente, desde el Capítulo 4 al 6, establecemos los fundamentos de la teoría de espacio de estados, donde veremos el modelamiento matemático, sus transformaciones y propiedades. Esta teoría nos permitirá programar las simulaciones y el diseño de una manera más real, ya que una de sus capacidades es la de poder trabajar con sistemas de múltiples entradas y salidas, cosa que no era posible con la teoría de control clásica. Del mismo modo, introduce los conceptos de controlabilidad y observabilidad. 
Los capítulos centrales de este texto son el 7 y el 8. Ahí es donde aplicamos todos los conocimientos previos para el desarrollo de controladores y observadores de estado.

Desde el Capítulo 9 al 10 veremos una serie de teorías complementarias que sirven para mejorar el comportamiento de los sistemas de control de estados. Hemos visto conveniente resaltar el seguimiento y el control óptimo. Todas estas teorías se utilizarán en las diferentes partes del análisis o diseño. Igualmente, no debemos limitarnos a ellas porque si sabemos emplear otras teorías que puedan apoyar a este campo, así como de otras nuevas que puedan desarrollarse, debemos experimentar su uso en la teoría de control moderno.

Adicionalmente, se presenta un apéndice donde planteamos una introducción al Matlab. Su finalidad es enseñar a usar este programa, sino de explicar algunas de sus funciones y aplicaciones para ayudar a su empleo en el control.

Los temas teóricos están presentados con ejemplos en su aplicación con la finalidad de una fácil y rápida comprensión, y casi en su totalidad son desarrollados adicionalmente en Matlab, siempre y cuando sea aplicable.

Enrique Arnáez Braschi 


\section{Capítulo 1: Introducción al control moderno}

A continuación, recordaremos una serie de conceptos que deberemos tener presentes para comprender los temas que se desarrollarán posteriormente.

Comenzamos por definir al control realimentado como una operación que, a pesar de estar bajo la influencia de perturbaciones, tiende a reducir la diferencia entre alguna entrada de referencia y el resultado del proceso esperado, y lo continúa haciendo con base en esta diferencia hasta que el sistema se estabilice y en el mejor de los casos, hasta que la diferencia desaparezca.

El sistema se define como una combinación de componentes que actúan conjuntamente y que cumplen determinado objetivo.

La planta es el objeto sobre el cual se ejecutan las acciones especiales organizadas con el fin de lograr resultados de funcionamiento deseados. Es el objeto que se desea controlar.

\section{Gráfico 1.1. Sistema}

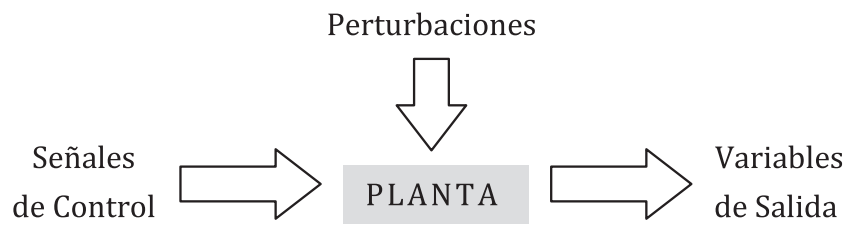

Las acciones elaboradas por el controlador se denominan señales de control, mientras que las que no dependen del sistema de control, que no se pueden predecir y que no son deseadas, se denominan perturbaciones.

Los resultados para los cuales se diseñó el controlador son las variables de salida.

El proceso es la sucesión de cambios que se producen en una planta: cambios de materia, energía, información, etcétera. 


\section{Gráfico 1.2. Sistema de control en varias formas}
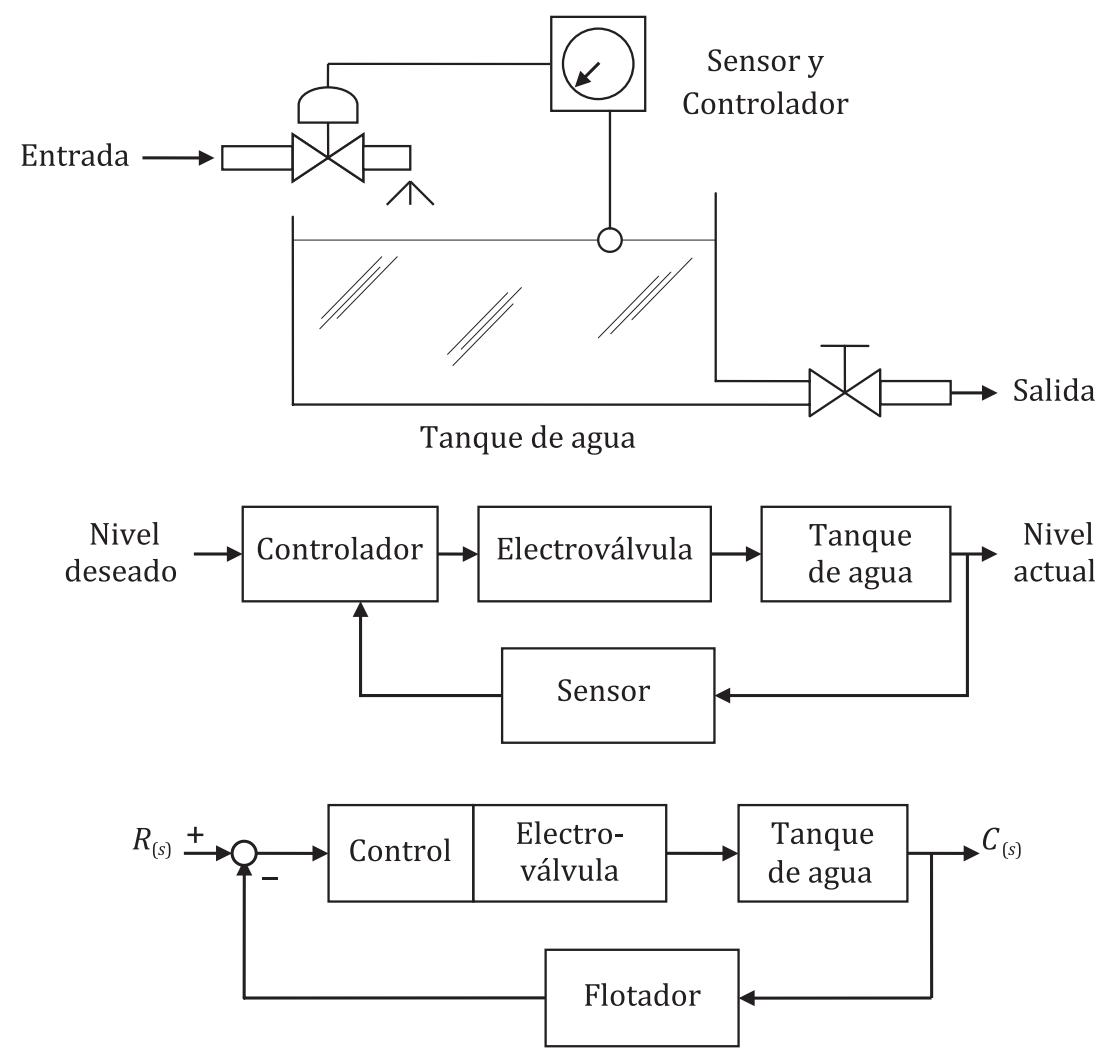

\subsection{Clasificación de los sistemas de control}

a. Según su dimensión:

- Sistemas de parámetros concentrados: representados por ecuaciones diferenciales u otra expresión que le dé carácter finito.

- Sistemas de parámetros distribuidos: son de carácter infinito por lo que requieren de derivadas parciales dentro de su representación, por ejemplo: el modelo de un oleoducto.

b. Según el conocimiento de sus parámetros:

- Sistemas determinísticos: sistemas de parámetros conocidos.

- Sistemas estocásticos: donde algunos o todos sus parámetros son conocidos probabilísticamente.

c. Según el tipo de continuidad:

- Sistemas continuos: tienen definición para todo instante de tiempo y mediante puntos contiguos, por ejemplo una parábola. 
- Sistemas discontinuos: tienen una o más definiciones en cada instante de tiempo las cuales no son, necesariamente, puntos contiguos, por ejemplo: un tren de pulsos o una tangente.

- Sistemas discretos: están definidos en ciertos periodos, por ejemplo: una señal muestreada.

d. Según su estructura matemática:

- Sistemas lineales: su comportamiento puede ser representado por una línea recta. Se puede aplicar el principio de superposición.

- Sistemas no lineales: no pueden ser representados por una línea recta en su totalidad o en una porción del mismo, así sea muy pequeña.

e. Según el comportamiento de sus parámetros:

- Sistemas invariantes en el tiempo: cuyos parámetros son iguales para todos los instantes de tiempo, por ejemplo: un sistema de suspensión mecánica.

- Sistemas variantes en el tiempo: cuyos parámetros cambian conforme va transcurriendo el tiempo, por ejemplo: la masa de un cohete o de un carro Fórmula 1, volumen de cuerpos sometidos a diferentes temperaturas y otros similares.

Para las aplicaciones en el curso que estamos desarrollando y por motivos de instrucción vamos a utilizar sistemas lineales invariantes en el tiempo.

\subsection{Transformadas de Laplace:}

Las Transformadas de Laplace se encargan de facilitar el cálculo de operaciones íntegro-diferenciales. Esto se realiza cambiando el dominio del tiempo a uno imaginario que denominamos Dominio de Laplace, en donde la solución de ecuaciones diferenciales se alcanza mediante procedimientos algebraicos.

Las Transformadas de Laplace más comunes son las siguientes:

$$
\begin{array}{rlrl}
\mathcal{L}\left\{\delta_{(t)}\right\}=1 & \mathcal{L}\{t\}=\frac{1}{s^{2}} \quad \mathcal{L}\left\{u_{(t)}\right\}=\frac{1}{s} \\
\mathcal{L}\left\{t^{n-1}\right\}=\frac{(n-1) !}{s^{n}} \quad \mathcal{L}\left\{e^{-a t}\right\}=\frac{1}{s+a} & \mathcal{L}\{\cos (a t)\}=\frac{s}{s^{2}+a^{2}} \\
\mathcal{L}\{\sin (a t)\}=\frac{a}{s^{2}+a^{2}} &
\end{array}
$$

Es conveniente recalcar que para realizar las transformaciones inversas de Laplace se utilizan las mismas fórmulas, pero en sentido contrario. 


\subsubsection{Propiedades de las Transformadas de Laplace:}

Las transformaciones se facilitan notablemente cuando, además de las fórmulas más comunes, aplicamos las propiedades que rigen a estas. A continuación vamos a presentar las más importantes:

$$
\mathcal{L}\left\{\frac{d^{n} f_{(t)}}{d t^{n}}\right\}=s^{n} F_{(s)}-s^{n-1} f_{\left(0^{+}\right)}-s^{n-2} \dot{f}_{\left(0^{+}\right)}-\ldots-f_{\left(0^{+}\right)}^{n-1}
$$

Donde todas las derivadas de la función son iguales a 0 , cuando las condiciones iniciales son 0 .

$$
\begin{aligned}
\mathcal{L}\left\{\int_{0}^{t} f_{(t)} d t\right\} & =\frac{F_{(s)}}{s} \\
\mathcal{L}\left\{e^{-a t} f_{(t)}\right\} & =F_{(s+a)} \\
\mathcal{L}\left\{f_{\left(\frac{t}{a}\right)}\right\} & =a F_{(a s)} \\
\left\{\int_{0}^{t} f_{1(t-\tau)} f_{2(\tau)} d \tau\right\} & =F_{1(s)} F_{2(s)}
\end{aligned}
$$

Teorema del valor inicial:

$$
f_{\left(0^{+}\right)}=\lim _{s \rightarrow \infty} s F_{(s)}
$$

Teorema del valor final:

$$
\lim _{t \rightarrow \infty} f_{(t)}=f_{(\infty)}=\lim _{s \rightarrow 0} s F_{(s)}
$$

\subsubsection{Expansión por fracciones parciales:}

La expansión por fracciones parciales es utilizada para descomponer una función que contiene polinomios en el numerador y en el denominador, en un conjunto de fracciones que respondan fácilmente a la transformación inversa de Laplace.

Los casos más frecuentes son expresiones con denominador compuesto por una multiplicación de binomios, y expresiones con denominador compuesto por binomios elevados a alguna potencia. Para los demás casos que no están explícitamente citados, se deberán combinar estas alternativas de solución y deberá usarse mucha álgebra para factorizar de la manera adecuada estas expresiones y nos apoyaremos bastante en las propiedades de las Transformadas de Laplace.

Presentaremos la forma de resolver estas expansiones con los siguientes ejemplos: 
Ejemplo E.1.1: Expandir en fracciones parciales y calcular la transformada inversa de Laplace de la siguiente función:

$$
F_{(s)}=\frac{s+4}{(s+1)(s+2)}
$$

\section{Solución:}

Expandamos la expresión anterior en la cantidad de fracciones iguales a los binomios que contenga el denominador y colocaremos cada binomio en el denominador de cada fracción, dejando como incógnita a cada numerador.

$$
F_{(s)}=\frac{s+4}{(s+1)(s+2)}=\frac{A}{s+1}+\frac{B}{s+2}
$$

Igualamos los numeradores para resolver las incógnitas mediante ecuaciones simultáneas,

$$
\begin{gathered}
s+4=A(s+2)+B(s+1) \\
s+4=A s+2 A+B s+B \\
s+4=(A+B) s+(2 A+B)
\end{gathered}
$$

igualamos los coeficientes:

$$
\begin{gathered}
A+B=1 \\
2 A+B=4
\end{gathered}
$$

restamos (1) de (2):

$$
A=3
$$

reemplazamos (3) en (1) y despejamos:

$$
B=-2
$$

reemplazamos estos resultados en la expansión de fracciones y obtenemos la respuesta:

$$
F_{(s)}=\frac{s+4}{(s+1)(s+2)}=\frac{3}{s+1}-\frac{2}{s+2}
$$

Para finalizar, ahora podemos aplicar la transformada inversa de Laplace:

$$
\mathcal{L}^{-1}\left\{\frac{3}{s+1}-\frac{2}{s+2}\right\}=3 e^{-t}-2 e^{-2 t}
$$

Ejemplo E.1.2: Expandir en fracciones parciales y calcular la transformada inversa de Laplace de la siguiente función:

$$
F_{(s)}=\frac{2 s+10}{s^{2}+2 s+5}
$$




\section{Solución:}

Debemos trabajar el denominador a manera de representar en el denominador funciones que sean fáciles de transformar y para ello utilizaremos las propiedades de las Transformadas de Laplace.

$$
F_{(s)}=\frac{2 s+10}{s^{2}+2 s+5}=\frac{2 s+10}{\left(s^{2}+2 s+1\right)+4}=\frac{2 s+10}{(s+1)^{2}+2^{2}}
$$

Una vez que hemos conseguido una forma adecuada en el denominador, separaremos las expresiones en dos sumandos utilizando los del numerador:

$$
\begin{gathered}
F_{(s)}=\frac{2 s+10}{(s+1)^{2}+2^{2}}=\frac{2 s+2}{(s+1)^{2}+2^{2}}+\frac{8}{(s+1)^{2}+2^{2}} \\
F_{(s)}=\frac{2 s+2}{(s+1)^{2}+2^{2}}+\frac{8}{(s+1)^{2}+2^{2}}=2 \frac{s+1}{(s+1)^{2}+2^{2}}+4 \frac{2}{(s+1)^{2}+2^{2}}
\end{gathered}
$$

Aplicando las propiedades, podemos agrupar:

$$
F_{(s)}=2 \frac{(s+1)}{(s+1)^{2}+2^{2}}+4 \frac{2}{(s+1)^{2}+2^{2}}
$$

Luego, mediante la transformada inversa de Laplace, tenemos:

$$
\begin{gathered}
\mathcal{L}^{-1}\left\{F_{(s)}\right\}=\mathcal{L}^{-1}\left\{2 \frac{(s+1)}{(s+1)^{2}+2^{2}}+4 \frac{2}{(s+1)^{2}+2^{2}}\right\} \\
\mathcal{L}^{-1}\left\{F_{(s)}\right\}=\mathcal{L}^{-1}\left\{2 \frac{(s+1)}{(s+1)^{2}+2^{2}}\right\}+\mathcal{L}^{-1}\left\{4 \frac{2}{(s+1)^{2}+2^{2}}\right\} \\
\mathcal{L}^{-1}\left\{\frac{2 s+10}{s^{2}+2 s+5}\right\}=2 e^{-t} \cos (2 t)+4 e^{-t} \sin (2 t)
\end{gathered}
$$

Ejemplo E.1.3: Expandir en fracciones parciales y calcular la transformada inversa de Laplace de la siguiente función:

$$
F_{(s)}=\frac{s^{2}+2 s+4}{(s+1)^{3}}
$$

\section{Solución:}

Deberemos expandir la expresión inicial en tantas fracciones como binomios contenga el denominador, colocando literales en los numeradores, las cuales serán las incógnitas por 
resolver. Es decir, si tenemos un binomio cubo, un binomio cuadrado y un binomio común, se deberán tener seis fracciones parciales.

$$
F_{(s)}=\frac{s^{2}+2 s+4}{(s+1)^{3}}=\frac{A}{s+1}+\frac{B}{(s+1)^{2}}+\frac{C}{(s+1)^{3}}
$$

Ahora, tal como en el ejemplo a, debemos igualar los numeradores:

$$
\begin{gathered}
s^{2}+2 s+4=A(s+1)^{2}+B(s+1)+C \\
s^{2}+2 s+4=A s^{2}+2 A s+A+B s+B+C \\
s^{2}+2 s+4=A s^{2}+(2 A+B) s+(A+B+C)
\end{gathered}
$$

Igualamos los coeficientes y resolvemos las ecuaciones simultáneas:

$$
\begin{gathered}
A=1 \\
2 A+B=2 \\
A+B+C=4
\end{gathered}
$$

Reemplazamos (1) en (2) y despejamos $B$ :

$$
B=0
$$

Reemplazamos (1) y (4) en (3) y despejamos C:

$$
C=3
$$

Con los valores de las variables literales, obtenemos las fracciones parciales:

$$
F_{(s)}=\frac{s^{2}+2 s+4}{(s+1)^{3}}=\frac{1}{s+1}+\frac{0}{(s+1)^{2}}+\frac{3}{(s+1)^{3}}=\frac{1}{s+1}+\frac{3}{(s+1)^{3}}
$$

y por último, aplicamos la transformada inversa de Laplace:

$$
\begin{gathered}
\mathcal{L}^{-1}\left\{F_{(s)}\right\}=\mathcal{L}^{-1}\left\{\frac{1}{s+1}+\frac{3}{(s+1)^{3}}\right\} \\
\mathcal{L}^{-1}\left\{F_{(s)}\right\}=\mathcal{L}^{-1}\left\{\frac{1}{s+1}\right\}=\mathcal{L}^{-1}\left\{\frac{3}{2} \frac{2}{(s+1)^{3}}\right\} \\
\mathcal{L}^{-1}\left\{F_{(s)}\right\}=e^{-t}+\frac{3}{2} t^{2} e^{-t} \\
\mathcal{L}^{-1}\left\{\frac{s^{2}+2 s+3}{(s+1)^{3}}\right\}=e^{-t}\left(1+\frac{3}{2} t^{2}\right)
\end{gathered}
$$


Para poder revisar todo el contenido de esta edición, visite nuestra tienda virtual.
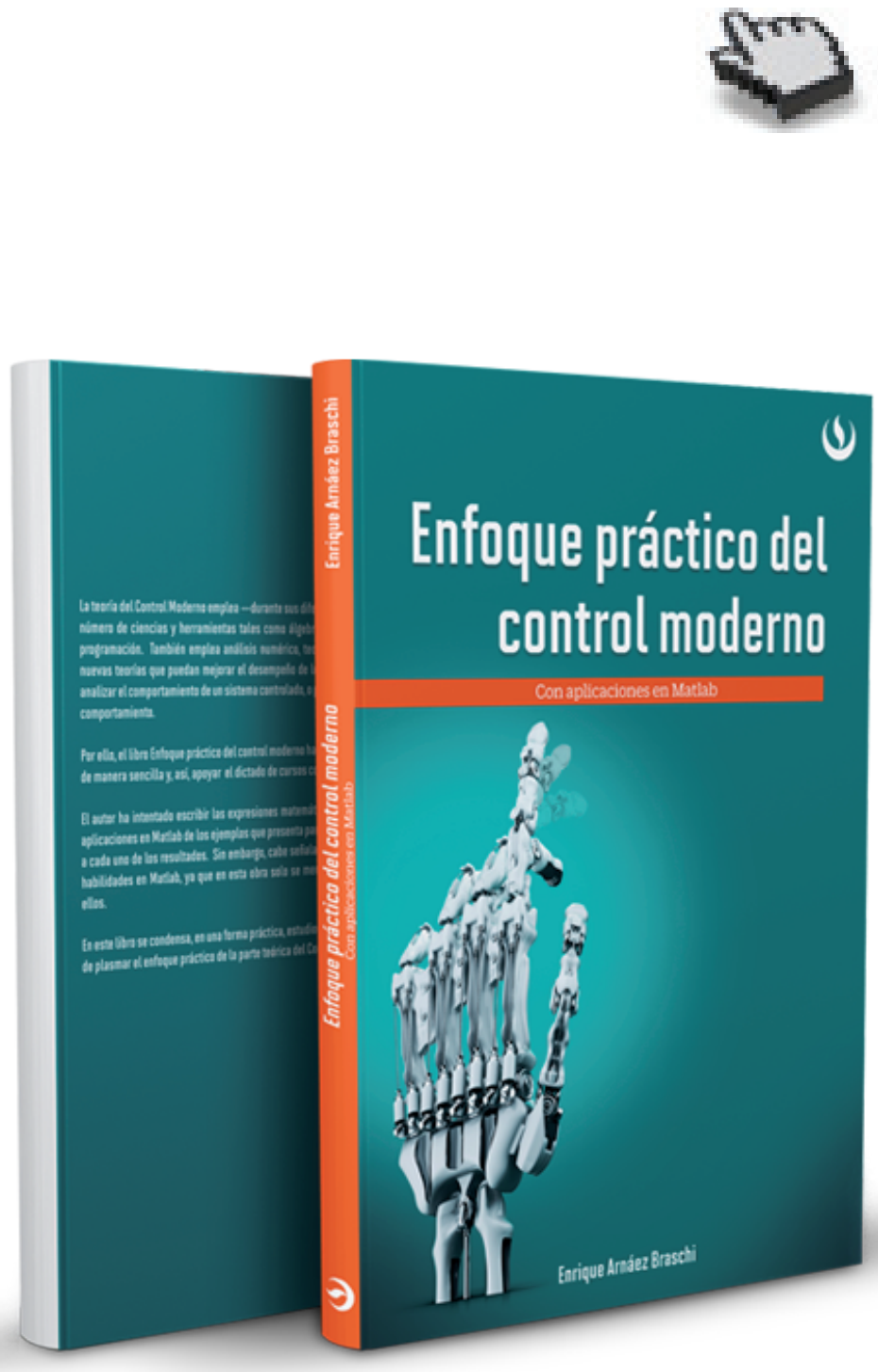